\section{Resultados a 10 años en trasplantes renales con donantes vivos no relacionados. Experiencia de 22 años}

\author{
CAROLINA CARMONA R. ${ }^{1}$, JUAN PABLO HUIDOBRO E. ${ }^{1}$, \\ JORGE VEGA S. ${ }^{1,2,3}$
}

\section{Survival among renal allograft recipients from non related living donors}

\begin{abstract}
Background: There is a gap between the number of patients requiring a renal allograft and the number of potential deceased donors (DD). One alternative is using allografts from non-related living donors (NRLD). Aim: To compare survival and complications of renal allograft recipients from $D D$, related living donors (RLD) and NRLD. Material and Methods: Observational study of a cohort of renal allograft recipients. Of 253 transplants performed in a Chilean region between 1981 and 2003, 20 patients received and allograft from a NRLD. Graft and patient survival of these patients were compared with those of $93 \mathrm{pa-}$ tients receiving an allograft from a related living donor and 140 receiving it from a DD. Patients were followed for 10 years or until death or dialysis requirement. Results: No significant differences between groups in graft and patient survival, deaths with a functioning graft or return to dialysis were observed. Receptors of DD had more hospital admissions during the first years after receiving the graft, usually due to infections. Also a delayed graft function was more common among them. Glomerular filtration rate ten years after the graft was similar among the three groups. Conclusions: No differences in graft or patient survival was observed between patients receiving a renal allograft from NRLD, RLD or DD.

(Rev Med Chile 2015; 143: 147-157)

Key words: Graft survival; Kidney transplantation; Living donors; Prognosis; Unrelated donors.
\end{abstract}

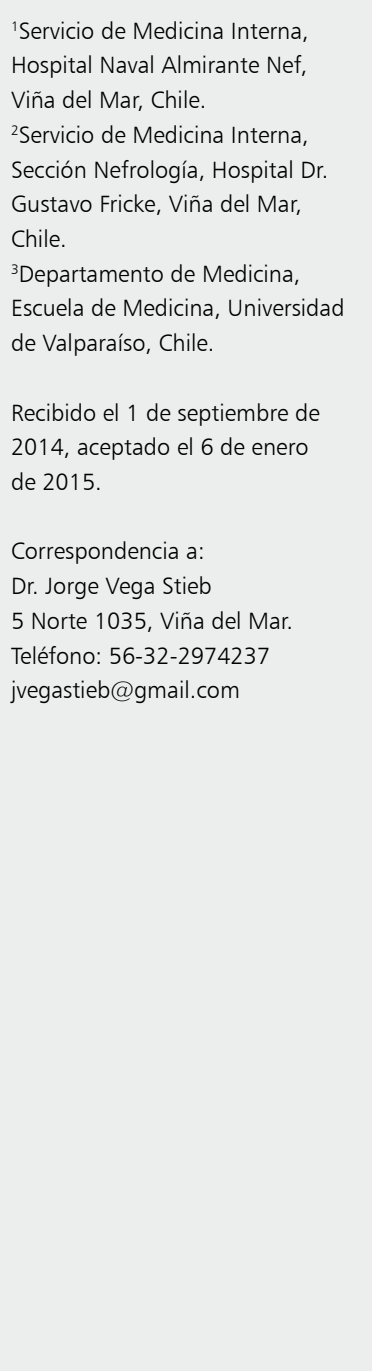

E 1 número de pacientes en diálisis crónica ha aumentado en los últimos años constituyendo un problema de salud pública con incremento en los costos de su atención. Los pacientes que esperan un trasplante renal (TXR) también han aumentado significativamente, superando la oferta de órganos de donantes fallecidos. Esto ha aumentado la mortalidad de pacientes en la lista de espera ${ }^{1-5}$. Ello ha motivado la búsqueda de nuevas fuentes de órganos como donantes marginales, con corazón parado y donantes vivos no relacionados (DVNR) $)^{5-9}$.

Los TXR con un donante vivo (DV) tienen numerosas ventajas respecto a los trasplantes de donante fallecido (DF), como el poder evitar la diálisis realizando el trasplante en forma anticipada y contar con una isquemia más breve previo a su implantación ${ }^{1,10-12}$. Los DV no están expuestos a la inestabilidad cardiovascular e isquemia de los riñones de DF durante el período que precede a la muerte cerebral ${ }^{7}$.

Los TXR con DVNR fueron frecuentemente efectuados en la década de 1960 cuando la diálisis no estaba universalmente disponible ${ }^{13}$. Con los años, al aumentar su disponibilidad, se redujeron los TXR con DVNR fundamentalmente por moti- 
vos éticos ${ }^{5}$. Salvo en las donaciones provenientes entre esposos, en las donaciones entre amigos no era fácil establecer que sólo estaba motivada por razones altruistas ni se podía descartar que existieran retribuciones económicas a cambio de la donación.

Los avances en inmunosupresión han llevado a una mejoría de los resultados de los TXR. En otros países se ha comunicado que los TXR con DVNR tienen buenos resultados a corto ${ }^{14,15} \mathrm{y}$ largo plazo ${ }^{16-19}$ con bajo riesgo para los donantes $^{20,21,22}$. La supervivencia de pacientes e injertos de los TXR con DVNR es similar a los con DVR y superiores a los trasplantes con $\mathrm{DF}^{16,17,22-26}$. Este tipo de donación se ha practicado con cautela para evitar el comercio de órganos, habitualmente con donantes emocionalmente relacionados (cónyuges) o altruistas en sistemas de donación cruzada y anónima.

El objetivo de este trabajo es conocer si la supervivencia de injertos y pacientes, así como sus complicaciones durante los primeros 10 años del TXR; fue diferente en los trasplantados renales con DVNR respecto a los trasplantados con DVR o con DF.

\section{Material y Método}

Realizamos un estudio observacional de cohorte retrospectiva que incluyó a los pacientes trasplantados entre abril de 1981 y el 31 de diciembre de 2003. Entre esas fechas se efectuaron 303 trasplantes renales en los Hospitales Naval Almirante Nef, Dr. Gustavo Fricke y Clínica Reñaca. Uno efectuado en Estados Unidos (USA) inició su seguimiento en uno de estos hospitales. De estos 304 injertos se incluyeron 253 en esta investigación. Los 51 restantes fueron excluidos por tratarse de menores de 18 años $(n=16)$, información insuficiente $(\mathrm{n}=11)$ y falla primaria del injerto $(\mathrm{n}=24)$. De estos últimos, 17 fueron TXR con DF, 6 con DVR y sólo uno con DVNR.

De los 253 trasplantes incluidos en esta investigación, 140 se efectuaron con DF, 93 con DVR (padres, hermanos, hijos, primos, tíos) y 20 con DVNR (esposos, amigos). Todas estas donaciones se efectuaron durante un período en que la ley de trasplantes chilena lo permitía.

Efectuamos una comparación de los TXR con DVNR con los efectuados con DVR y con DF. El seguimiento de los receptores fue 10 años desde la fecha del TXR, a menos que hubieran fallecido con un riñón funcionante o regresado a diálisis crónica.

Se registraron los datos demográficos, tiempo de permanencia en diálisis previo al TXR, datos inmunológicos (incompatibilidades HLA A, B, DR; tasa de anticuerpos contra linfocitos), drogas inmunosupresoras utilizadas, función del injerto con los años, complicaciones y estado del paciente al final del seguimiento.

La velocidad de filtración glomerular se estimó mediante la fórmula MDRD ${ }^{27}$.

Para el análisis de las variables categóricas y continuas se utilizaron el test exacto de Fisher y Kruskall-Wallis, respectivamente. El análisis de supervivencia de los injertos y de pacientes en los 3 grupos se efectuó con la técnica Kaplan-Meier, evaluando su asociación mediante el test de logrank. Se consideró como diferencias significativas cuando $\mathrm{p}<0,05$.

\section{Resultados}

Los donantes de los receptores de un injerto de DF fueron mayoritariamente hombres y de menor edad que los DVR y DVNR. Los receptores de injertos provenientes de un DVR fueron más jóvenes (Tabla 1).

Los receptores de un riñón de un DF permanecieron en diálisis crónica un período significativamente más prolongado que los receptores de un injerto de un DV. No hubo diferencias entre los tres grupos en la existencia previa al TXR de diabetes mellitus, ni en el antecedente de injertos renales previos. La tasa máxima histórica de anticuerpos contra un panel de linfocitos (PRA) o la tasa previa a la realización del trasplante, no fue diferente entre los grupos. Las incompatibilidades en los antígenos DR fueron significativamente menores en las parejas de trasplantados con DF. Los receptores de un DVNR tuvieron más incompatibilidades en los antígenos HLA A y B (Tabla 1).

La proporción de pacientes que tuvieron una función retardada del injerto fue mayor en los sujetos que recibieron un injerto desde un DF, y también lo fue la concentración sérica de creatinina al momento del alta después de la operación (Tablas 2 y 3). La velocidad de filtración glome- 
Trasplantes renales con donantes vivos no relacionados - C. Carmona et al

rular estimada (eVFG) durante los primeros dos años del TXR fue menor en los trasplantados con un DF, sin embargo, no fue diferente en los 8 años siguientes (Tabla 3 ). No hubo diferencias durante los tres primeros años del TXR en la prevalencia de hematuria ni en el uso de más de dos drogas hipotensoras, sin embargo, la prevalencia de proteinuria $>500 \mathrm{mg} / 24$ horas a los tres años del TXR fue menor en los trasplantados con DF (Tabla 3).

El número de reoperaciones y de hospitalizaciones durante el primer año del TXR fue mayor en los trasplantados con DF. Las hospitalizaciones originadas por infecciones durante el primer año fueron también más frecuentes en estos pacientes. En cambio, las hospitalizaciones por causas cardiovasculares no fueron diferentes entre los tres grupos en los primeros 10 años del TXR. La aparición de una neoplasia maligna durante los primeros 10 años del TXR no fue distinta entre los tres grupos como tampoco lo fue la incidencia de infecciones virales, tuberculosis, fracturas, episodios de gota aguda, eritrocitosis y daño hepático crónico. La aparición de diabetes mellitus post trasplante fue más frecuente en los que recibieron un riñón desde DF y DVNR (Tabla 2).
La proporción de pacientes que recibieron terapia para un rechazo agudo durante los primeros 10 años del TXR fue menor en los receptores de un riñón de DF que en los que lo recibieron de donantes vivos, relacionados y no relacionados (Tabla 4). No hubo diferencias entre los grupos en la proporción de pacientes a los que se les efectuó una biopsia renal durante el primer año del TXR. En los años siguientes los que recibieron un injerto de un DVR fueron biopsiados con mayor frecuencia (Tabla 4). Los receptores de un riñón desde un DF recibieron un esquema inmunosupresor que incluyó anti-calcineurínicos con mayor frecuencia que los receptores de otro tipo de donante, durante los 10 primeros años de trasplante (Tabla 4).

La proporción de pacientes que regresaron a diálisis crónica o que fallecieron con un injerto funcionante no fue diferente entre los tres grupos (Tablas 3). Las infecciones fueron la causa más frecuente de fallecimientos con un injerto funcionante (Tabla 5).

La supervivencia de injertos y de pacientes durante los primeros 10 años del TXR fue similar en los tres grupos de trasplantados (Figura 1 y 2 ).

Tabla 1. Características de donantes y receptores en 253 trasplantados renales con tres diferentes tipos de donantes

\begin{tabular}{|c|c|c|c|c|}
\hline & $\begin{array}{l}\text { Trasplantes } \\
\text { con donante } \\
\text { vivo no } \\
\text { relacionado } \\
(n=20)\end{array}$ & $\begin{array}{l}\text { Trasplantes } \\
\text { con donante } \\
\text { vivo } \\
\text { relacionado } \\
(n=93)\end{array}$ & $\begin{array}{c}\text { Trasplantes } \\
\text { con donante } \\
\text { fallecido } \\
(n=140)\end{array}$ & $\mathbf{p}$ \\
\hline Donante masculino, n (\%) & $10(50)$ & $43(46,2)$ & $87(64,4)$ & 0,02 \\
\hline Edad del donante, mediana (RIC) & $38(30-52)$ & $36(29-46)$ & $31(22-44)$ & 0,01 \\
\hline Receptor masculino, n (\%) & $16(80)$ & $48(51,6)$ & $80(57,1)$ & NS \\
\hline Edad del receptor (años), mediana (RIC) & $45(35-51)$ & $32(24-40)$ & $42(33-51)$ & 0,0001 \\
\hline Número de incompatibilidades HLA A y B, mediana (RIC) & $3(2-3)$ & $2(1-2)$ & $2(1-2)$ & 0,0001 \\
\hline Número de incompatibilidades HLA DR, mediana (RIC) & $1(1-2)$ & $1(0-1)$ & $0(0-1)$ & 0,01 \\
\hline \% de PRA previo al TXR, mediana (RIC) & $0(0-3)$ & $0(0-0)$ & $0(0-5)$ & NS \\
\hline \% de PRA histórico máximo, mediana (RIC) & $3(0-16)$ & $3(0-13)$ & $6(0-15)$ & NS \\
\hline Intervalo (meses) entre inicio de HD y TXR, mediana (RIC) & $15(11-22)$ & $12(7-21)$ & $42(23-65)$ & 0,0001 \\
\hline Diabetes mellitus previa al TXR, n (\%) & $1(5)$ & $3(3,2)$ & $6(4,3)$ & NS \\
\hline
\end{tabular}

TXR: trasplante renal; HLA: antígenos de histocompatibilidad; PRA: anticuerpos reactivos contra linfocitos de panel; RIC: rango intercuartil; NS: no significativo. 
Tabla 2. Complicaciones en 253 trasplantados renales con tres diferentes tipos de donantes

\begin{tabular}{|c|c|c|c|c|}
\hline & $\begin{array}{l}\text { Trasplante } \\
\text { con donante } \\
\text { vivo no } \\
\text { relacionado } \\
(n=20)\end{array}$ & $\begin{array}{l}\text { Trasplante } \\
\text { con donante } \\
\text { vivo } \\
\text { relacionado } \\
(n=93)\end{array}$ & $\begin{array}{c}\text { Trasplante } \\
\text { con donante } \\
\text { fallecido } \\
(n=140)\end{array}$ & $\mathbf{p}$ \\
\hline Función renal retardada, n (\%) & $2(10)$ & $12(13)$ & $47(34,3)$ & $<0,001$ \\
\hline Reintervención quirúrgica en post operatorio, n (\%) & $2(10)$ & $7(7,5)$ & $17(12,4)$ & NS \\
\hline Reoperación durante en el primer año del TXR, n (\%) & $1(5)$ & $1(1,1)$ & $15(10,7)$ & 0,007 \\
\hline $\begin{array}{l}\text { Reoperación durante el segundo o tercer año del TXR, } \\
\mathrm{n}(\%)\end{array}$ & 0 & $4(4,6)$ & $4(3,1)$ & NS \\
\hline Hospitalizaciones durante primer año del TXR, n (\%) & $9(45)$ & $48(52,2)$ & $94(67,1)$ & 0,003 \\
\hline Hospitalizaciones durante $2^{\circ}$ y $3^{\circ}$ del TXR, n (\%) & $7(20)$ & $39(45,9)$ & $63(49,2)$ & NS \\
\hline Hospitalizaciones año 4 en adelante del TXR, n (\%) & $10(55,6)$ & $58(76,3)$ & $87(75,5)$ & NS \\
\hline $\begin{array}{l}\text { Hospitalizaciones por infecciones durante el primer año } \\
\text { del TXR, } n(\%)\end{array}$ & $4(20)$ & $19(20,7)$ & $49(35)$ & 0,05 \\
\hline $\begin{array}{l}\text { Hospitalizaciones por infecciones durante los primeros } \\
5 \text { años del TXR, n (\%) }\end{array}$ & $7(35)$ & $36(38,7)$ & $60(42,9)$ & NS \\
\hline $\begin{array}{l}\text { Hospitalizaciones por infecciones después del año } 5 \text { del } \\
\text { TXR, } n(\%)\end{array}$ & $6(35,3)$ & $19(28,8 \%)$ & $41(39,8)$ & NS \\
\hline $\begin{array}{l}\text { Hospitalizaciones por causa cardiovascular durante el } \\
\text { primer año del TXR, n (\%) }\end{array}$ & $1(5)$ & $6(6,6 \%)$ & $14(10)$ & NS \\
\hline $\begin{array}{l}\text { Hospitalizaciones por causa cardiovascular en los pri- } \\
\text { meros } 5 \text { años del TXR, n (\%) }\end{array}$ & 1 (5) & $18(19,4)$ & $30(21,4)$ & NS \\
\hline $\begin{array}{l}\text { Hospitalizaciones por causa cardiovascular después del } \\
5^{\circ} \text { año del TXR, n (\%) }\end{array}$ & $2(11,8)$ & $4(6,1)$ & $10(9,7)$ & NS \\
\hline $\begin{array}{l}\text { Neoplasias malignas en los primeros } 5 \text { años del TXR, } \\
\text { n (\%) }\end{array}$ & 1 (5) & $3(3,2)$ & $10(7,1)$ & NS \\
\hline $\begin{array}{l}\text { Neoplasias malignas después de los primeros } 5 \text { años del } \\
\text { TXR, } n(\%)\end{array}$ & $2(11,8)$ & $4(6,1)$ & $13(12,6)$ & NS \\
\hline Diabetes mellitus post TXR, n (\%) & $4(20)$ & $2(2,2)$ & $18(12,9)$ & 0,002 \\
\hline Daño hepático crónico, n (\%) & $4(20)$ & $5(5,5)$ & $9(6,5)$ & NS \\
\hline Eritrocitosis post TXR, n (\%) & $4(20)$ & $10(11)$ & $23(16,6)$ & NS \\
\hline Herpes zoster, n (\%) & $5(25)$ & $12(13,2)$ & $17(12,2)$ & NS \\
\hline Infección por CMV, n (\%) & $1(5)$ & $6(6,6)$ & $8(5,8)$ & NS \\
\hline Tuberculosis, n (\%) & 0 & $2(2,2)$ & $4(2,9)$ & NS \\
\hline Fracturas óseas, n (\%) & $3(15)$ & $7 \quad(7,7)$ & $5(3,6)$ & NS \\
\hline Necrosis óseas avasculares, n (\%) & $1(5)$ & $8(8,8)$ & $7(5)$ & NS \\
\hline Crisis de gota aguda, n (\%) & $2(10)$ & $1(5,5)$ & $12(8,6)$ & NS \\
\hline
\end{tabular}

TXR: trasplante renal, NS: no significativo, CMV: citomegalovirus. 
Trasplantes renales con donantes vivos no relacionados - C. Carmona et al

\section{Tabla 3. Evolución del trasplante renal con los años en 253 trasplantados renales con 3 diferentes tipos de donantes}

\begin{tabular}{|c|c|c|c|c|c|}
\hline & $\begin{array}{r}\text { Tras } \\
\text { con o } \\
\text { vil } \\
\text { relac } \\
\text { (n }\end{array}$ & $\begin{array}{l}\text { plante } \\
\text { donante } \\
\text { vo no } \\
\text { cionado } \\
=20 \text { ) }\end{array}$ & $\begin{array}{l}\text { Trasplante } \\
\text { con donante } \\
\text { vivo } \\
\text { relacionado } \\
(n=93)\end{array}$ & $\begin{array}{l}\text { Trasplante } \\
\text { con donante } \\
\text { fallecido } \\
(n=140)\end{array}$ & $\mathbf{p}$ \\
\hline Creatininemia al alta (mg/dL), mediana (RIC) & 1,3 & $(1-2)$ & $1,2(1-2)$ & $1,6(1-2)$ & 0,0001 \\
\hline eVFG al año del TXR (ml/min/1,73 m²), mediana (RIC) & 64 & $(51-80)$ & $62,5(49-83)$ & $54,5(41-67)$ & 0,001 \\
\hline eVFG a 2 años del TXR (ml/min/1,73 m²), mediana (RIC) & 63 & $(43-80)$ & $60 \quad(48-81)$ & $54 \quad(47-64)$ & 0,04 \\
\hline eVFG a 3 años del TXR (ml/min/1,73 m²), mediana (RIC) & 60,5 & $(52-71)$ & $55 \quad(42-80)$ & $51,5(43-63)$ & NS \\
\hline eVFG a 5 años del TXR (ml/min/1,73 m²), mediana (RIC) & 58,5 & $(45-70)$ & $56 \quad(43-67)$ & $49 \quad(40-63)$ & NS \\
\hline eVFG a 10 años del TXR (ml/min/1,73 m²), mediana (RIC) & 51,5 & $(34-55)$ & $47 \quad(36-57)$ & $50 \quad(37-64)$ & NS \\
\hline Hematuria al año del TXR, n (\%) & 1 & $(5,6)$ & $7 \quad(9,7)$ & $12 \quad(10,6)$ & NS \\
\hline Hematuria al año 2 del TXR, n (\%) & 1 & $(5,6)$ & $11(16,4)$ & $20 \quad(19,2)$ & NS \\
\hline Hematuria al año 3 del TXR, n (\%) & 2 & $(12,5)$ & $5 \quad(7,8)$ & $13 \quad(13)$ & NS \\
\hline Proteinuria > 500 mg/día al año del TXR, n (\%) & 1 & $(5,6)$ & $6(8,2)$ & $7 \quad(6,2)$ & NS \\
\hline Proteinuria > 500 mg/día al año 2 del TXR, n (\%) & 3 & $(17,7)$ & $6 \quad(9)$ & $7 \quad(6,7)$ & NS \\
\hline Proteinuria > 500 mg/día al año 3 del TXR, n (\%) & 3 & $(17,7)$ & $10 \quad(15,6)$ & $3(3)$ & 0,005 \\
\hline Uso de > 2 hipotensores al año del TXR, n (\%) & 2 & $(11,1)$ & $16 \quad(21,1)$ & $19(16,6)$ & NS \\
\hline Uso de $>2$ hipotensores al año 2 del TXR, n (\%) & 3 & $(16,67)$ & $12 \quad(17,9)$ & $24 \quad(22,9)$ & NS \\
\hline Uso de $>2$ hipotensores al año 3 del TXR, n (\%) & 4 & $(23,5)$ & $10 \quad(15,6)$ & $28 \quad(27,5)$ & NS \\
\hline Pacientes con TXR funcionante a la fecha de corte, n (\%) & 12 & $(60)$ & $57 \quad(61,2)$ & $83(59,3)$ & NS \\
\hline $\begin{array}{l}\left.\text { Última eVFG (ml/min/1,73 } \mathrm{m}^{2}\right) \text { en pacientes con TXR funcio- } \\
\text { nante a la fecha de corte, mediana (RIC) }\end{array}$ & 50 & $(34-64)$ & $47 \quad(36-58)$ & $50 \quad(39-64)$ & NS \\
\hline Pacientes que fallecieron con TXR funcionante, n (\%) & 2 & $(10)$ & $14 \quad(15)$ & $30 \quad(21,4)$ & NS \\
\hline $\begin{array}{l}\left.\text { Última eVFG ( } \mathrm{ml} / \mathrm{min} / 1,73 \mathrm{~m}^{2}\right) \text { en pacientes que fallecieron } \\
\text { con TXR funcionante, mediana (RIC) }\end{array}$ & 32,5 & $(16-49)$ & $37,5(23,5-56)$ & $41 \quad(24-62)$ & NS \\
\hline $\begin{array}{l}\text { Duración (meses) del TXR en pacientes que fallecieron con } \\
\text { TXR funcionante, mediana (RIC) }\end{array}$ & 72 & $(15-83)$ & $49 \quad(12-92)$ & $56 \quad(19-86)$ & NS \\
\hline Edad al fallecimiento (años) con TXR funcionante, mediana (RIC) & 50 & $(35-51)$ & $50 \quad(43-57)$ & $53,5(46-59)$ & NS \\
\hline Pacientes que regresaron a diálisis, n (\%) & 6 & (30) & $22 \quad(23,6)$ & $27 \quad(19,3)$ & NS \\
\hline $\begin{array}{l}\text { Duración del TXR en pacientes que regresaron a HDC, } \\
\text { (meses) mediana (RIC) }\end{array}$ & 90 & $(47-94)$ & $(20-72)$ & $23(12-69)$ & NS \\
\hline
\end{tabular}

eVFG: velocidad de filtración glomerular estimada; TXR: trasplante renal; HDC: hemodiálisis, RIC: rango intercuartil; NS: no significativo. 
Tabla 4. Inmunosupresión utilizada en 253 trasplantados renales con 3 diferentes tipos de donantes

\begin{tabular}{|c|c|c|c|c|}
\hline & $\begin{array}{l}\text { Trasplante } \\
\text { con donante } \\
\text { vivo no } \\
\text { relacionado } \\
(n=20)\end{array}$ & $\begin{array}{l}\text { Trasplante } \\
\text { con donante } \\
\text { vivo } \\
\text { relacionado } \\
(n=93)\end{array}$ & $\begin{array}{l}\text { Trasplante } \\
\text { con donante } \\
\text { fallecido } \\
(n=140)\end{array}$ & $\mathbf{p}$ \\
\hline $\begin{array}{l}\text { - Sin anti-calcineurínicos, n (\%) } \\
\text { - Con anti-calcineurínicos, n (\%) }\end{array}$ & $\begin{array}{rr}7 & (35 \%) \\
13 & (65 \%)\end{array}$ & $\begin{array}{ll}53 & (57 \%) \\
40 & (43 \%)\end{array}$ & $\begin{array}{r}20(14,3 \%) \\
120(85,7 \%)\end{array}$ & $<0,001$ \\
\hline $\begin{array}{l}\text { Inducción con anticuerpos, n (\%) } \\
\text { Uso de anticuerpos en TAR, n (\%) }\end{array}$ & $(5 \%)$ & $\begin{array}{ll}0 & \\
3 & (3,2 \%)\end{array}$ & $\begin{array}{l}2(1,4 \%) \\
8(5,8 \%)\end{array}$ & $\begin{array}{l}\text { NS } \\
\text { NS }\end{array}$ \\
\hline $\begin{array}{l}\text { TAR durante post-operatorio, } \mathrm{n}(\%) \\
\text { TAR durante primer año, } \mathrm{n}(\%) \\
\text { TAR entre año } 2^{\circ} \text { y } 5^{\circ} \text { año de TXR, } \mathrm{n}(\%) \\
\text { TAR año } 6 \text { en adelante, } \mathrm{n}(\%)\end{array}$ & $\begin{array}{ll}6 & (30 \%) \\
7 & (35 \%) \\
5 & (25 \%) \\
2 & (11,8 \%)\end{array}$ & $\begin{aligned} 27 & (29,4 \%) \\
38 & (40,9 \%) \\
29 & (30 \%) \\
9 & (14,1 \%)\end{aligned}$ & $\begin{array}{c}24(17,7 \%) \\
30(21,4 \%) \\
18(14,2 \%) \\
4(3,9 \%)\end{array}$ & $\begin{array}{l}\text { NS } \\
0,04 \\
0,004 \\
0,04\end{array}$ \\
\hline $\begin{array}{l}\text { MPDN durante } 1 \text { er año (gramos), mediana (RIC) } \\
\text { MPDN años } 2 \text { y } 3 \text { (gramos), mediana (RIC) }\end{array}$ & $\begin{array}{lr}0,5 & (0-2) \\
0 & (0-2)\end{array}$ & $\begin{array}{lr}1,5 & (0-3) \\
0 & (0-2)\end{array}$ & $\begin{array}{l}0(0-2) \\
0(0-0)\end{array}$ & $\begin{array}{l}0,007 \\
\text { NS }\end{array}$ \\
\hline $\begin{array}{l}\text { Biopsia renal durante } 1^{\text {er }} \text { año, } n(\%) \\
\text { Biopsia renal durante toda la evolución del TXR, n (\%) }\end{array}$ & $\begin{array}{ll}2 & (10 \%) \\
6 & (30 \%)\end{array}$ & $\begin{array}{ll}18 & (19,6 \%) \\
46 & (50 \%)\end{array}$ & $\begin{array}{l}26(18,7 \%) \\
45(32,6 \%)\end{array}$ & $\begin{array}{c}\text { NS } \\
0,02\end{array}$ \\
\hline $\begin{array}{l}\text { Inmunosupresión al año del TXR } \\
\text { - Sin anti-calcineurínicos, n (\%) } \\
\text { - Con anti-calcineurínicos, n (\%) }\end{array}$ & $\begin{array}{rr}8 & (40 \%) \\
12 & (60 \%)\end{array}$ & $\begin{array}{ll}45 & (49,5 \%) \\
46 & (50,5 \%)\end{array}$ & $\begin{array}{c}13(9,7 \%) \\
121(90,3 \%)\end{array}$ & $<0,001$ \\
\hline $\begin{array}{l}\text { Último esquema de inmunosupresión } \\
\text { - Sin anti-calcineurínicos, n (\%) } \\
\text { - Con anti-calcineurínicos, n (\%) }\end{array}$ & $\begin{array}{rr}6 & (30 \%) \\
14 & (70 \%)\end{array}$ & $\begin{array}{l}(47,8 \%) \\
(52,2 \%)\end{array}$ & $\begin{array}{c}12(8,9 \%) \\
123(91,1 \%)\end{array}$ & $<0,001$ \\
\hline
\end{tabular}

TAR: terapia anti rechazo; MPDN: metilprednisolona; TXR: trasplante renal; NS: no significativo.

Tabla 5. Causas de muerte con injerto funcionante en $\mathbf{4 6}$ trasplantados renales con $\mathbf{3}$ diferentes tipos de donantes

\begin{tabular}{|c|c|c|c|c|}
\hline & $\begin{array}{l}\text { Trasplante con } \\
\text { donante vivo } \\
\text { no relacionado } \\
(n=2)\end{array}$ & $\begin{array}{l}\text { Trasplante con } \\
\text { donante vivo } \\
\text { relacionado } \\
(n=14)\end{array}$ & $\begin{array}{l}\text { Trasplante con } \\
\text { donante } \\
\text { fallecido } \\
(\mathbf{n}=\mathbf{3 0})\end{array}$ & $\mathbf{p}$ \\
\hline Infecciones, n (\%) & $1(50 \%)$ & $6(43 \%)$ & $16(53,4 \%)$ & NS \\
\hline - Sepsis & 0 & 3 & 12 & \\
\hline - Neumonía & 0 & 3 & 2 & \\
\hline - Encefalitis & 0 & 0 & 1 & \\
\hline - Colitis pseudomembranosa por CD & 1 & 0 & 0 & \\
\hline - Empiema pleural & 0 & 0 & 1 & \\
\hline Enfermedades digestivas, $\mathrm{n}(\%)$ & $1(50 \%)$ & 0 & $6(20 \%)$ & NS \\
\hline - Daño Hepático/Hepatitis fulminante & 1 & 0 & 3 & \\
\hline - Pancreatitis aguda & 0 & 0 & 2 & \\
\hline - Diarrea crónica & 0 & 0 & 1 & \\
\hline Enfermedades cardiovasculares, n (\%) & 0 & $4(28,5 \%)$ & $4(13,3 \%)$ & NS \\
\hline - Edema pulmonar agudo & 0 & 2 & 1 & \\
\hline - Infarto agudo al miocardio & 0 & 0 & 1 & \\
\hline - Accidentes cerebrovasculares & 0 & 1 & 2 & \\
\hline - Trombosis mesentérica & 0 & 1 & 0 & \\
\hline Cáncer, n (\%) & 0 & $4(28,5 \%)$ & $4(13,3 \%)$ & NS \\
\hline
\end{tabular}

CD: Clostridium difficile. 


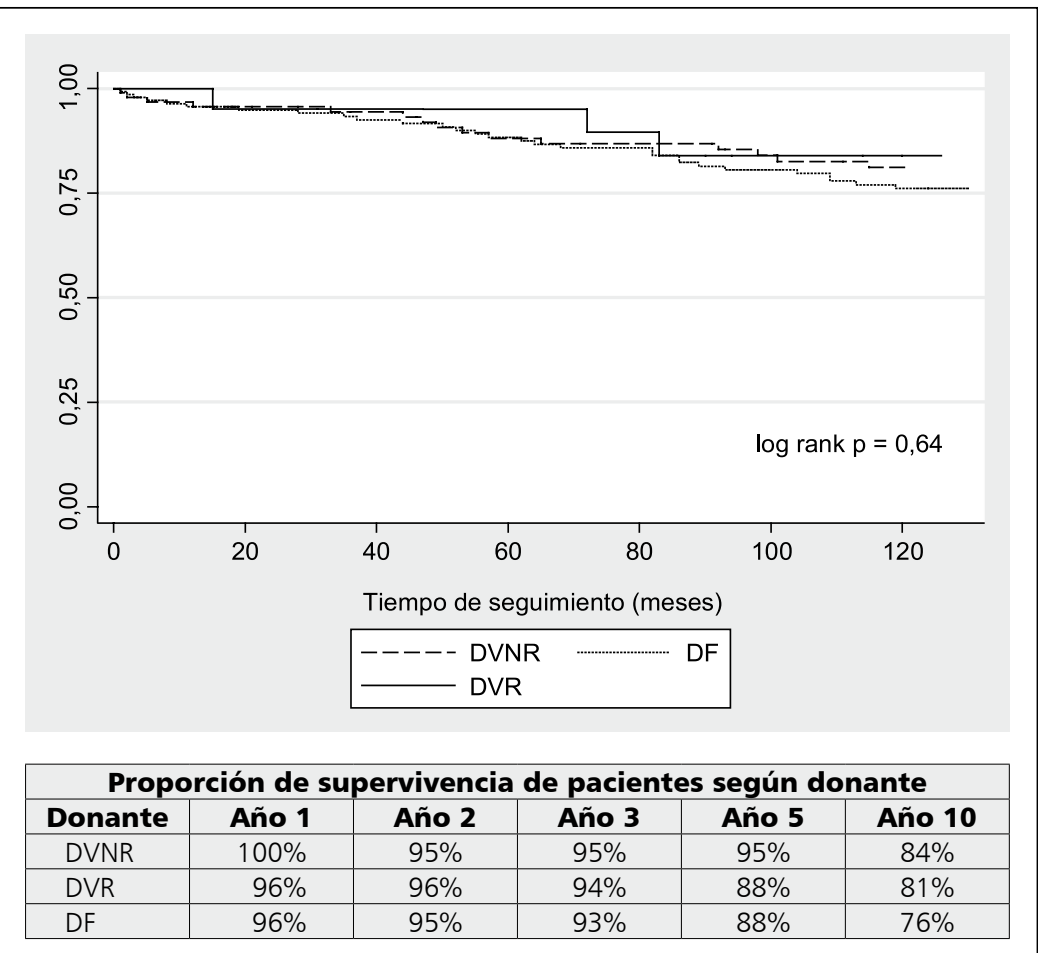

Figura 1. Curva de supervivencia de pacientes según donante (Kaplan-Meier). * Los pacientes que volvieron a diálisis fueron censurados. DVNR: donante vivo no relacionado; DVR: donante vivo relacionado; DF: donante fallecido.

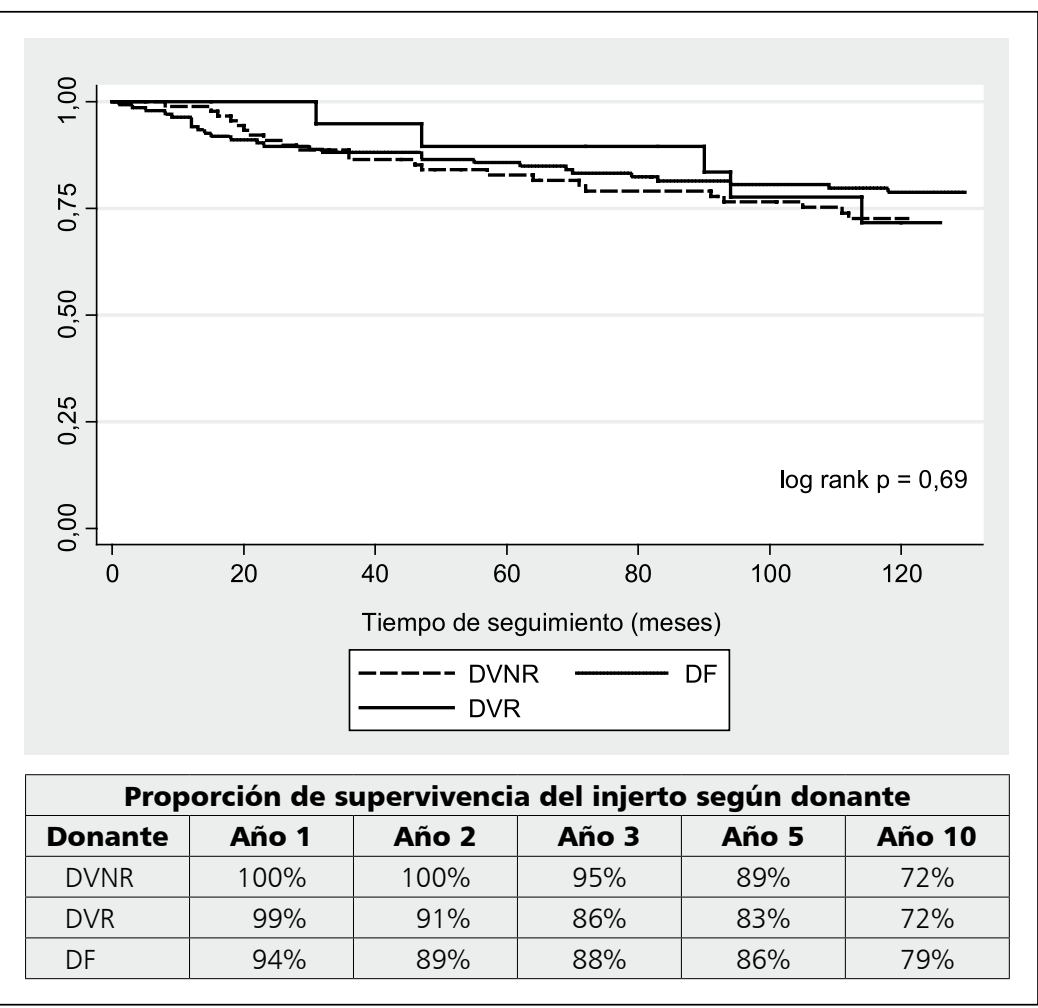

Figura 2. Curva de supervivencia del injerto según donante (KaplanMeier). *Los pacientes que fallecieron con un riñón funcionante fueron censurados. DVNR: donante vivo no relacionado; DVR: donante vivo relacionado; DF: donante fallecido. 


\section{Discusión}

En esta serie de 253 TXR realizados entre 1981 y 2003, 113 recibieron un injerto desde un DV y sólo 20 (18\%) de ellos recibieron un TXR de DVNR. Esta proporción es inferior a las comunicadas en otras series. En el registro nacional japonés de trasplante renal de 2009 se reportaron 1.123 TXR de DV y de estos 35\% fue de DVNR (cónyuges) ${ }^{28}$. En una serie publicada por Chkhotua et al el año 2003, de 129 TXR de DV, 38\% se efectuaron con un DVNR, siendo en su mayoría cónyuges $(51 \%)^{5}$. En el estudio publicado por Ahmad et al el año 2008 de 322 TXR con DV, 261 provinieron de DVR y $61(23,2 \%)$ DVNR $^{29}$.

En nuestra serie los receptores de un riñón de un DF permanecieron en diálisis crónica un período significativamente más prolongado que los receptores de un injerto de un DV (mediana de 42 meses), lo que es explicado por la escasez de órganos de donantes fallecidos. Vivek et al el año 2012 en su comunicación de los resultados a largo plazo de los TXR en India, mostró que la mediana de duración de la permanencia en diálisis fue de 9 a 12 meses $^{3}$.

El hallazgo en nuestra serie de que los donantes fallecidos fueron más jóvenes y más frecuentemente varones que los donantes vivos (relacionados o no), es comprensible, dado que una proporción importante de ellos fallecieron en accidentes.

Estudios realizados en Italia e India mostraron que el número de incompatibilidades HLA fue mayor en los TXR con DVNR que en los con DVR, similar a lo encontrado en nuestros resultados ${ }^{3,4}$.

La velocidad de filtración glomerular (VFG) a los 10 años del TXR en nuestros tres grupos de trasplantados no fue diferente. Ello es similar a lo reportado en otras series ${ }^{2,29}$.

En este estudio, la proporción de pacientes que recibieron terapia para un rechazo agudo del trasplante en el post-operatorio fue similar entre los tres grupos, semejante a lo encontrado por Voiculescu et $\mathrm{al}^{30}$. Fuller et al encontraron que los TXR con DVNR tenían tasas más altas de rechazos durante el primer año del trasplante que los efectuados con donantes relacionados, pero ello no influía en la supervivencia final del injerto ${ }^{31}$. Campbell mostró que los TXR con DV tenían una tasa superior de rechazos que los TXR con DF, sin embargo, estos presentaban una mayor supervivencia de pacientes e injertos ${ }^{32}$. En nuestra serie tuvimos una menor frecuencia de rechazos agudos en los receptores de DF en los primeros 10 años del TXR. Ello probablemente se debe a que estos pacientes recibieron con mayor frecuencia terapia inmunosupresora triasociada desde el momento del TXR.

La proporción de pacientes de nuestra serie que tuvieron una función retardada del injerto fue mayor en los que recibieron un injerto desde un DF (34\%) que los que lo recibieron de DVNR $(10 \%)$. Esto es una ventaja de este tipo de donantes ya que se conoce que la aparición de función retardada del injerto reduce la sobrevida a largo plazo de los injertos ${ }^{33}$. Ello fue similar a lo comunicado por Gjertson et al, quienes también encontraron esta complicación más frecuentemente en los TXR con DF (24\%) que en los TXR con DVNR (7\% $)^{22}$.

Hace algunas décadas, la tasa de supervivencia de injertos y pacientes en los TXR era más baja y las complicaciones más frecuentes que en la actualidad. Ello ha ido mejorando significativamente en los últimos años gracias al advenimiento de mejores esquemas inmunosupresores, el mejor manejo de las enfermedades infecciosas y los progresos en los cuidados postoperatorios del receptor ${ }^{2,8}$. Hariharan et al analizaron los resultados de 93.934 TXR realizados en USA entre el año 1988 y 1996 donde evidenciaron una mejoría sustancial de la supervivencia a corto y mediano plazo de los injertos, tanto en TXR de DV como $\mathrm{DF}^{34}$.

Dada la escasez creciente de órganos de DF, ha aumentado la proporción de TXR con DV. Su uso, inicialmente restringido por las potenciales complicaciones en el donante asociadas a la nefrectomía clásica, ha aumentado con la introducción de la nefrectomía laparoscópica, la que tiene una baja tasa de complicaciones $(0,1 \% \text { y } 0,03 \%)^{3,25,35}$.

Un estudio publicado por Najarian et al en 1992 mostró que durante el seguimiento a largo plazo de los sujetos que fueron donantes de un riñón para trasplante vivo, no hubo una mayor frecuencia de insuficiencia renal crónica que en el grupo control, como tampoco de otras complicaciones $^{20}$. Similares hallazgos fueron reportados por Fehrman-Ekholm et $\mathrm{al}^{21}$.

Los resultados del TXR con DV son habitualmente superiores a los con DF. Ellos proporcionan al receptor una mejor calidad de vida a largo pla$\mathrm{zo}^{36,37}$. Humar et al mostraron que la supervivencia de los TXR de DV a 8 años era de $62 \%$ en los años 60 y $87 \%$ en la década del $90^{16}$. Esta mejoría en los 
Trasplantes renales con donantes vivos no relacionados - C. Carmona et al

resultados también ha sido comunicada en otros centros $^{18,19}$.

A fines de la década de 1990 reapareció el entusiasmo por efectuar TXR con DVNR a raíz de la publicación de Terasaki et al donde comunicaron que estos pacientes tenían tasas similares de supervivencia a los trasplantados con DVR ${ }^{17}$. Ello ya había sido comunicado previamente en otras series $^{14,15}$. La experiencia de la Universidad de Wisconsin en 243 TXR de DVNR mostró resultados a largo plazo similares a los trasplantes haploidénticos (trasplantados que comparten un haplotipo) y superiores a los trasplantes con $\mathrm{DF}^{38}$. Ello ha hecho que los TXR con DVNR hayan llegado a ser una buena alternativa cuando no existen DVR ${ }^{5}$.

En nuestra serie, la supervivencia de injertos y de pacientes así como sus complicaciones durante los primeros 10 años del TXR fue similar en los 2 grupos (DVR y DVNR), semejante a lo encontrado por Simforoosh et al en una experiencia en 2.155 TXR entre el año 1984 y 2004. En ella encontró que no hubo diferencias en la supervivencia de pacientes e injertos a 1, 3, 5, 10 y 15 años en TXR con DVR y DVNR ${ }^{1}$. En un estudio Italiano que reportó una experiencia de 23 años de TXR de DV, no encontró diferencias significativas en la supervivencia ni del injerto ni del paciente, según el tipo de donante ${ }^{4}$. Centros de trasplante coreanos comunicaron una supervivencia a 5 años cercana al $80 \%$ en los TXR con DVNR, lo que fue similar a los TXR con vivos relacionados ${ }^{39,40}$.

Futagawa et al hicieron una revisión de la experiencia de varios centros en el registro UNOS entre 1998 y 2003 y observaron que en tres patologías específicas (glomeruloeslerosis focal y segmentaria, riñones poliquísticos y diabetes mellitus tipo 1) los trasplantados con DVNR tenían mejor supervivencia de injertos que los trasplantados con DVR provenientes de padres o hijos ${ }^{41}$.

A diferencia de otras publicaciones, en nuestro estudio la supervivencia de pacientes e injertos provenientes de DF fue similar a la de los que tuvieron un DV. Ello probablemente se explica porque los receptores de injertos provenientes de DF recibieron con mayor frecuencia un esquema inmunosupresor más potente que incluyó un fármaco anti-calcineurínico. En la primera década de los trasplantes comunicados en esta serie, el 80\% correspondió a donantes vivos (mayoritariamente relacionados). Estos recibieron habitualmente como esquema inmunosupresor la asociación de azatioprina y esteroides. Estos pacientes presentaron con mayor frecuencia crisis de rechazo agudo, situación que indudablemente influyó en la evolución del injerto. En las décadas siguientes, las donaciones provenientes de donantes fallecidos fueron la regla y mayoritariamente recibieron un esquema inmunosupresor más potente (triasociado) que incluyó un inhibidor de la calcineurina (ciclosporina). Fuggle et al también comunicó que la supervivencia a 1 y 5 años de los pacientes fue similar en los que recibieron un injerto de un DV como de un $\mathrm{DF}^{7}$ y Park et al encontraron que la supervivencia de los injertos con DF y DVNR a 1,5 y 10 años fue también semejante ${ }^{42}$.

Dado los buenos resultados comunicados en diversas series con los TXR con DVNR, esta modalidad se ha convertido en una alternativa útil para disminuir la lista de espera de quienes esperan un órgano de un DF y poder así reservar estos para los pacientes que no tienen ninguna otra alternativa de donantes.

El comercio de órganos es un factor importante en este tipo de TXR que ha limitado su uso ${ }^{29}$. En Chile, la actual ley de trasplante no permite este tipo de donación con excepción de esposos o parejas, en los que la eventualidad de una donación motivada por razones comerciales no existe. Esto no ocurre en otros países en desarrollo en los que existe el tráfico comercial de órganos ${ }^{43,44}$. Para evitar esta situación, las donaciones provenientes de sujetos no relacionados deben ser aprobadas por los comités de ética de los hospitales y por el Ministerio de Salud, después de una evaluación psicosocial completa verificando que la donación sea voluntaria y no económicamente compensada. De todas maneras es difícil de garantizar en 100\% que las donaciones sean absolutamente altruistas.

En varios países desarrollados se están empleando donantes vivos no relacionados en sistemas de donación cruzada incluyendo en ellos a donantes voluntarios altruistas. Esto ha permitido reducir las listas de espera de pacientes que aguardan por un órgano de sujetos fallecidos y ha permitido tener injertos de muy buena calidad ${ }^{45-48}$.

En suma, en nuestra serie, los que recibieron un injerto desde un DVNR tuvieron una supervivencia de injertos y pacientes similar a la de los que recibieron un riñón de un pariente consanguíneo o de un donante fallecido, y no tuvieron una tasa mayor de complicaciones en los primeros 10 años de seguimiento. 


\section{Referencias}

1. Simforoosh N, Basiri A, Fattahi MR, Einollahi B, Firouzan A Pour-Reza-Gholi F, et al. Living unrelated versus living related kidney transplantation: 20 years' experience with 2155 cases. Transplant Proc. 2006; 38: 422-5.

2. Chkhotua AB, Klein T, Shabtai E, Yussim A, Bar-Nathan $\mathrm{N}$, Shaharabani E, et al. Kidney transplantation from living-unrelated donors: comparison of outcome with living-related and cadaveric transplants under current immunosuppressive protocols._Urology. 2003; 62: 10026.

3. Vivek B, Pankaj R, Aruna V, Manoj R, Kamal R, Himanshu $\mathrm{V}$, et al. Long-term outcomes of renal transplants from spousal and living-related and other living-unrelated donors: a single center experience. J Assoc Physicians India. 2012; 60: 24-7.

4. Santori G, Barocci S, Fontana I, Bertocchi M, Tagliamacco A, Biticchi R, et al. Kidney transplantation from living donors genetically related or unrelated to the recipients: a single-center analysis. Transplant Proc. 2012; 44: 1892-6.

5. Chkhotua AB, Klein T, Shabtai EL, Yussim A, BarNathan N, Shaharabani E, et al. Kidney transplantation from living donors: comparison of results between related and unrelated donor transplants under new immunosuppressive protocols._Isr Med Assoc J. 2003; 5: 622-5.

6. D'Alessandro AM, Sollinger HW, Knechtle SJ, Kalayoglu M, Kisken WA, Uehling DT, et al. A 28-year Living related and unrelated donors for kidney transplantation experience. Ann Surg. 1995; 222: 353-62; discussion 3624.

7. Fuggle SV, Allen JE, Johnson RJ, Collett D, Mason PD, Dudley C, et al. Factors affecting graft and patient survival after live donor kidney transplantation in the UK. Transplantation. 2010; 89: 694-701.

8. Kwon OJ, Kim YH, Ahn BK, Kang CM, Kwak JY. Longterm graft outcome of living donor renal transplantation: single center experience. Transplant Proc. 2005; 37: 690-2 (2-3).

9. Park YH, Min SK, Lee JN, Lee HH, Jung WK, Lee JS, et al. Risk factors on graft survival of living donor kidney transplantation._Transplant Proc. 2004; 36: 2023-5.

10. Binet I, Bock AH, Vogelbach P, Gasser T, Kiss A, Brunner F, et al. Outcome in emotionally related living kidney donor transplantation. Nephrol Dial Transplant 1997; 12: 1940-8.

11. Roodnat JI, van Riemsdijk IC, Mulder PG, Doxiadis I, Claas FH, IJzermans JN, et al. The superior results of living-donor renal transplantation are not completely caused by selection or short cold ischemia time: A single-center, multivariate analysis. Transplantation 2003; 75: 2014.

12. Bos EM, Leuvenink HG, van Goor H, Ploeg RJ. Kidney grafts from brain dead donors: Inferior quality or opportunity for improvement? Kidney Int 2007; 72: 797.

13. Kuss R. Human renal transplant memories, 1951 to 1981. In: Terasaki PI ed, History of transplantation: 35 Years Recollection. Los Angeles: UCLA Tissue Typing Laboratory, 1991: 39.

14. Levey AS, Hou S, Bush HL. Kidney transplantation from unrelated living donors, time to reclaim a discarded opportunity. N Engl J Med 1986; 314: 914-6.

15. Cortesini R, Berloco P, Famulari A. Long term results in recipients of mismatched related and unrelated living kidneys in the cyclosporine age. Transplant Proc 1988; 20: 41-2.

16. Humar A, Durand B, Gillingham K, Payne WD, Sutherland DE, Matas AJ. Living unrelated donors in kidney transplants: Better long-term results than with nonHLA-identical living related donors? Transplantation 2000; 69: 1942.

17. Terasaki PI, Cecka JM, Gjertson DW. High survival rates of kidney transplants from spousal and living unrelated donors. N Engl J Med 1995; 333: 333.

18. Jakobsen A, Living renal transplantation-the Oslo experience. Nephrol Dial Transplant 1997; 12: 1825-7. 6.

19. Matas AJ, Payne WD, Sutherland DE, Humar A, Gruessner RW, Kandaswamy R, et al. 12,500 Living donor kidney transplants: a single-center experience. Ann Surg 2001; 234: 149-64.

20. Najarian JS, Chavers BM, McHugh, Matas AJ. 20 years or more of follow-up of living kidney donors. Lancet 1992; 240: 807-10.

21. Fehrman-Ekholm I, Elinder CG, Stenbeck M, Tyden G, Groth CG. Kidney donors live longer. Transplantation 1997; 64: 976-8.

22. Gjertson DW, Cecka JM. Living unrelated donor kidney transplantation. Kidney Int. 2000; 58: 491-9.

23. Cecka JM. The UNOS scientific renal transplant registry. Clin Trasplant 1996; 1-14.

24. Lowell JA, Brennan DC, Shenoy S, Hagerty D, Miller S, Ceriotti C, et al. Living unrelated renal transplantation provides comparable results to living-related renal transplantation: a 12-year single-center experience. Surgery. 1996; 119: 538-43.

25. D'Alessandro AM, Pirsch JD, Knechtle SJ, Odorico JS, Van der Werf WJ, Collins BH, et al. Living unrelated renal donation: the University of Wisconsin experience. Surgery. 1998; 124: 604-10; discussion 610-1.

26. Cecka JM. Kidney Transplantation form living unrelated donors. Annu Rev Med 2000; 51: 393-406. 
Trasplantes renales con donantes vivos no relacionados - C. Carmona et al

27. Levey AS, Bosch JP, Lewis JB, Greene T, Rogers N, Roth D. A more accurate accurate method to estimate glomerular filtration rate from serum creatinine: a new prediction equation. Modification of Diet in Renal Disease Study Group Ann Intern Med. 1999; 130 (6): 461-70.

28. Ishikawa N, Yagisawa T, Sakuma Y, Fujiwara T, Kimura T, Nukui A, et al. Kidney transplantation of living unrelated donor-recipient combinations._Transplant Proc. 2012; 44: 254-6.

29. Ahmad N, Ahmed K, Khan MS, Calder F, Mamode N, Taylor Y, et al. Living-unrelated donor renal transplantation: an alternative to living-related donor transplantation? Ann R Coll Surg Engl 2008; 90: 247-50.

30. Voiculescu A, Ivens K, Hetzel GR, Hollenbeck M, Sandmann W, Grabitz K, et al. Kidney transplantation from related and unrelated living donors in a single German centre. Nephrol Dial Transplant. 2003; 18: 418-25.

31. Fuller TF, Feng S, Brennan TV, Tomlanovich S, Bostrom A, Freise CE. Increased rejection in living unrelated versus living related kidney transplants does not affect short-term function and survival. Transplantation. 2004; 78: 1030-5.

32. Campbell SB, Hothersall E, Preston J, Brown AM, Hawley CM, Wall D, et al. Frequency and severity of acute rejection in live- versus cadaveric-donor renal transplants. Transplantation. 2003; 76: 1452-7.

33. Piñón J, Bueno J, Esparza E, Gómez A, Rodríguez E, Álvarez C. Impacto de la función renal retardada en la sobrevida del injerto renal de donante cadáver. Rev Med Inst Mex Seguro Soc 2009; 47 (1): 33-8.

34. Hariharan S, Johnson CP, Bresnahan BA, Taranto SE, McIntosch MJ, Stablein D. Improved graft survival after renal transplantation in the United States, 1988 to 1996. N Engl J Med 2000; 342: 605-12.

35. Jacobs SC, Cho E, Dunkin BJ, Flowers JL, Schweitzer E, Cangro C, et al. Laparoscopic live donor nephrectomy: the University of Maryland 3-year experience. J Urol 2000; 164: 1494-9.

36. Liem YS, Weimar W. Early living-donor kidney transplantation: A re- view of the associated survival benefit. Transplantation 2009; 87: 317.

37. Innocenti GR, Wadei HM, Prieto M, Dean PG, Ramos EJ, Textor S, et al. Preemptive living donor kidney transplantation: Do the benefits extend to all recipients? Trans- plantation 2007; 83: 144.

38. Shames BD, D'Alessandro AM, Pirsch JD. Living-unrelated renal transplantation at the University of Wisconsin. In: Cecka JM, Terasaki PI, eds. Clinical transplants 2001. Los Angeles, UCLA Immunogenetics Center 2002, p. 149.

39. Park K, Kim YS, Lee EM, Lee HY, Han DS. Single-center experience of unrelated living-donor renal transplantation in the cyclosporine era, in Clinical Transplants 1992, edited by Terasaki, Cecka JM, Los Angeles, UCLA Tissue Typing Laboratory, 10. Pre-transplant transfusions 1993, pp 249-56.

40. Yoon YS, Jin DC, Yang CW, Kim SY, Bang BK, Kim W, et al. The effect of HLA mismatching on graft survival in living donor kidney transplants: Catholic Medical Center. 1984 to 1993, edited by Terasaki PI, Cecka JM, 13. Los Angeles, UCLA Tissue Typing Laboratory, 1994, pp 275-83.

41. Futagawa Y, Waki K, Gjertson DW, Terasaki PI. Livingunrelated donors yield higher graft survival rates than parental donors._Transplantation. 2005; 79: 1169-74.

42. Park YH, Min SK, Lee JN, Lee HH, Jung WK, Lee JS, et al. Comparison of survival probabilities for livingunrelated versus cadaveric renal transplant recipients. Transplant Proc. 2004; 36: 2020-2.

43. Thiel G. Emotionally related living kidney donation: pro and contra. Nephrol Dial Transplant 1997; 12: 1820-4.

44. Chugh KS, Jha V. Commerce in transplantation in Third World countries. Kidney Int 1996; 49: 1181-6.

45. Rees M, Kopke JE, Pelletier RP, Segev D, Rutter ME, Fabrega AJ, et al. A nonsimultaneous, extended, altruisticdonor chain. N Engl J Med 2009; 630: 1096-101.

46. Roth AE, Sönmez T, Unver MU, Delmonico FL, Saidman SL. Utilizing list exchange and nondirected donation trough "chain" paired kidney donations. Am J Transplant 2006; 6: 2694-705.

47. Matas AJ, Garvey CA, Jacobs CL, Kahn JP. Nondirected donation of kidneys from living donors. N Engl J Med 2000; 343: 433-6.

48. Montgomery RA, Katznelson S, Bry WI, Sachary AA Houp J, Hiller JM, et al. Successful three-way kidney paired donation with cross-country live donor allograft transport. Am J Transplant 2008; 8: 2163-8. 